\title{
Teoria da resposta ao item aplicado ao inventário de vida diária de Katz numa amostra do Hospital Regional da Asa Norte - Brasília/DF
}

\author{
Daniel Portela de Deus Albano*
}

\section{Resumo}

Neste trabalho, será analisado o Inventário de Vida Diária de Katz (AVD KATZ) a partir da análise estatística da Teoria de Resposta ao Item (TRI) (q). Alguns estudos mostram que a demência é ligada a uma susceptibilidade genética e causa danos às atividades funcionais do indivíduo e, por isso, a importância da avaliação funcional do idoso. Para tanto, existem as Avaliações de Vida Diária que medem as funções de autonomia básica do indivíduo. A TRI consegue substituir, em partes, a Teoria Clássica dos Testes (TCT), pois avalia se o item realmente mede o traço latente que deveria medir. Os objetivos do estudo foram: analisar se os itens do AVD Katz realmente mediam autonomia; analisar as respostas de pacientes e verificar a discriminação e a dificuldade de cada item. A amostra total recolhida do Hospital Regional da Asa Norte (HRAN) de Brasília/DF, foi de 67 pacientes, sendo $42(62,7 \%)$ mulheres e 25 $(37,3 \%)$ homens. A partir das análises feitas com o software Xcalibre, pode-se notar que o teste AVD Katz tem uma boa sensibilidade em diferenciar pacientes com declínio cognitivo e início de processo demencial, de acordo com a pontuação do Clinical Dementia Rating (CDR). Além disso, essa proposta mostrou-se como um teste que mede a autonomia do paciente em relação às suas atividades de vida e seus itens têm boa correlação com o total do teste. O teste tem, atualmente, boa validade, porém, futuramente, com uma melhor educação e o advento da tecnologia ao alcance de todos, alguns itens poderão sofrer modificações.

Palavras-chave: Avaliação funcional. Demência. Teoria de resposta ao item (TRI). Inventário de atividades diárias.

\section{Introdução}

O envelhecimento populacional é um fenômeno que vem sendo observado há algum tempo pelo mundo e começa a fazer parte dos desafios da saúde pública contemporânea (VERAS et al., 1987). Era notório em países desenvolvidos no começo do Século XX e, a partir do fim desse mesmo Século, passou a ser

* Possui graduação em Psicologia pelo Centro Universitário de Brasília (2009), especialização em Neuropsicologia pelo Instituto Brasileiro de Neuropsicologia e Ciências Cognitivas e mestrado em Psicologia pelo Centro Universitário de Brasília (2014). E-mail: portela@live.com.

$\rightarrow$ http://dx.doi.org/10.5335/rbceh.2014.3910

Recebido em: 01/04/2014. Aprovado em: 10/06/2014 
uma realidade brasileira (PACHECO; SANTOS, 2004).

Segundo o censo de 2010, realizado pelo Instituto Brasileiro de Geografia e Estatística (IBGE), o Brasil possui mais de 14 milhões de pessoas acima de 65 anos de idade $(7,4 \%$ do total populacional). Em meados de 2010, o país foi posicionado em sexto lugar no ranking de países com grande número de idosos (SCHOUERI JÚNIOR et al., 2000). Projeções recentes indicam que esse segmento passará a $15 \%$ em 2020 (FREITAS, 2004) e mais de 31 milhões de idosos até o ano 2025 (CORAZZA, 2001).

A consequência mais provável do envelhecimento é que haja, num futuro próximo, um aumento no número de casos de demência, a qual é compreendida como um declínio cognitivo e/ ou comportamental de caráter crônico e, geralmente, progressivo. Causa restrições graduais nas atividades diárias (higiene, cuidados de casa, família, etc.) e que não pode ser explicado por modificações da consciência, na mobilidade ou na capacidade sensorial (deficiências visuais, auditivas, etc.). Existem mais de setenta tipos de demência, algumas com sua etiologia bem definida, outras nem tanto, como a Demência de Alzheimer (DA) (CAIXETA, 2007).

A Demência se coloca entre uma das maiores causas de morbidade entre os idosos e sua prevalência está entre $2 \%$ e $25 \%$ em idosos com 65 anos ou mais (FRATIGLIONI et al., 1991). A Demência de Alzheimer (DA) é o tipo mais comum no idoso, afetando pelo menos $5 \%$ dos indivíduos com mais de 65 anos e $20 \%$ dos idosos com mais de 80 anos (JORM; JACOMB, 1987).
Estudos recentes, como o de Andel et al. (2008), mostram que o risco de demência é parcialmente determinado por uma susceptibilidade genética individual, e que o envolvimento ativo nas atividades mentais, físicas e sociais pode retardar o aparecimento da enfermidade, preservando as funções cognitivas. Nesse contexto, é inserida a ideia de avaliação funcional em que se busca verificar em que nível as doenças ou os agravos impedem o desempenho de atividades cotidianas dos idosos de forma autônoma e independente, ou seja, sem o auxílio de outras pessoas ou de adaptações, permitindo um desenvolvimento de um planejamento assistencial mais adequado (DUARTE et al., 2007).

A avaliação funcional pode ser definida como uma tentativa sistematizada de medir os níveis nos quais a pessoa é capaz de desempenhar determinadas atividades ou funções em diferentes áreas, utilizando-se das habilidades diversas para o desempenho das tarefas da vida cotidiana, para a realização de interações sociais, em suas atividades de lazer e em outros comportamentos do dia a dia. De modo geral, representa uma maneira de medir se uma pessoa é ou não capaz de desempenhar as atividades necessárias para cuidar de si mesma independentemente e, caso não seja, verificar se essa necessidade de ajuda é parcial ou em menor grau (WILKINS et al., 2001).

A perda da capacidade funcional leva à incapacidade de realização de atividades de vida diária (AVD) e nas atividades instrumentais de vida diária (AIVD). A primeira refere-se às atividades de cuidados pessoais básicos (ir ao banheiro, vestir-se, locomover-se, etc.), 
já a segunda, refere-se às atividades mais complexas (fazer compras, usar o telefone, cozinhar, etc.) (OKUMA, 1998).

Vários instrumentos foram criados para avaliar as AVD e AIVD, porém muitos são genéricos e voltados para doenças específicas, fazendo com que os itens sejam os mais variados possíveis. Os testes variam entre número de itens, amplitude de mensuração, dificuldade de item, tipo de medida que cada escala adota, rigor psicométrico, etc. (MCHORNEY; COHEN, 2000). Essas grandes variações entre os instrumentos os deixam impossíveis para comparação de dados. Por exemplo, indivíduos que completam o AVD Katz não podem ser comparados com aqueles que completam o AIVD Lawton, que aborda os itens citados no parágrafo anterior, (LAWTON; BRODY, 1969) e assim por diante.

Neste trabalho, foi escolhido o AVD KATZ, uma escala que reflete em seis funções psicosociobiológicas: higienização, ato de vestir-se, uso do banheiro, locomoção, continência e alimentação (KATZ; AKPOM, 1976). Katz e Stroud (1989) mostraram que a recuperação do desempenho funcional dessas seis atividades básicas da vida cotidiana de idosos incapacitados era semelhante à sequência observada no processo de desenvolvimento de uma criança. Também verificaram, por meio de estudos antropológicos, a existência de similaridade entre essas seis funções e o comportamento nas sociedades primitivas, o que sugeria que eram biológica e psicossocialmente primárias, refletindo uma hierarquização das respostas neurológicas e locomotoras. Os itens são pontuados em zero para dependência total, 0,5 para dependência parcial (necessita ajuda para realização de tarefas) e um para autonomia total (SCAZUFCA, 2002) e são separados por: a) higiene; b) vestir-se; c) uso de banheiro; d) locomoção; e) continência; f) alimentação.

Para avaliarmos os dados, resolvemos utilizar a Teoria da Resposta ao Item (TRI) que é uma alternativa à Teoria Clássica dos Testes, antiga Psicometria, na qual se pode fazer análise de parâmetros de itens, avaliando se o item (que no caso é o comportamento do indivíduo) realmente mede o traço latente (que seria a causa última deste comportamento), inclusive conhecida como Teoria do Traço Latente (PASQUALI, 2007). Dentre esses parâmetros estão a dificuldade do item (ou preferência categórica, em testes de aptidão ou personalidade) e a discriminação. Além de ser uma medida não linear, a TRI supre uma fidedignidade estimada para cada item (SPECTOR; FLEISHMAN, 1998) e não somente ao teste como um todo.

Hambleton, Swaminathan e Rogers (1991) apresentaram cinco grandes avanços que a TRI trouxe: o cálculo do nível de aptidão do sujeito independe da amostra de itens utilizados; a avaliação dos parâmetros dos itens (dificuldade e discriminação) independe da amostra de sujeitos utilizada; a possibilidade de emparelhar itens com a aptidão do sujeito (itens mais fáceis para sujeitos com habilidades inferiores e mais difíceis para os mais aptos); a não obrigatoriedade em fazer suposições que aparentam ser improváveis; e o fato de não ser necessário o trabalho com testes estritamente paralelos.

Com o crescente volume de pesquisas sobre novos instrumentos de avaliação 
da capacidade funcional, é necessário realizar uma revisão dos testes utilizados nas redes pública e particular para que possa ser verificada a validade desses com a contemporaneidade. Algumas escalas ajudam a mensurar o quanto a capacidade funcional está prejudicada ou preservada (KAALOLA et al., 2006).

O AVD Katz, apesar de ser um teste relativamente antigo, continua sendo usado em larga escala nos ambulatórios e clínicas no Brasil e em outros países. A partir desse pensamento, alguns questionamentos devem ser levantados, a respeito de sua validade e de formas de aplicação, bem como sua relação preditiva de comportamentos que indicam algum tipo de enfermidade, como as próprias demências citadas anteriormente.

Tendo levantado essas dúvidas pertinentes, definem-se alguns objetivos para o estudo: analisar se os itens do AVD Katz realmente medem a autonomia do sujeito; verificar as respostas de pacientes, considerando, assim, a discriminação e a dificuldade, e analisar os resultados para adaptação na realidade atual.

\section{Metodologia}

\section{Sujeitos}

A amostra total foi de 67 indivíduos, sendo $42(62,7 \%)$ mulheres e $25(37,3 \%)$ homens, todos com idade entre 65 e 90 anos. Não foram excluídos do estudo indivíduos com outras queixas associadas, tais como hipertensão, diabetes, etc. Os dados levantados constam desde 2004 até 2009 . O projeto foi aprovado pelo Comitê de Ética em Pesquisa da Secretaria da Saúde de Brasília, protocolo número
122/08, para que fosse possível a realização desse. A distribuição da escolaridade pode ser vista na Tabela 1.

Tabela 1 - Distribuição da escolaridade da amostra coletada.

\begin{tabular}{l|r}
\multicolumn{1}{c|}{ Escolaridade da Amostra } & \multicolumn{1}{c}{ N (\%) } \\
\hline Analfabetos & $25(37,3)$ \\
Nível Fundamental Incompleto & $31(46,3)$ \\
Nível Fundamental Completo & $4(6,0)$ \\
Nível Médio Incompleto & $3(4,4)$ \\
Nível Médio Completo & $4(6,0)$ \\
Nível Superior & $4(6,0)$ \\
Total & $67(100,0)$ \\
\hline
\end{tabular}

\section{Softwares}

Para a calibragem dos itens foi utilizado o software Xcalibre 4.1 (Assessment Systems Corporation) e para as análises estatísticas o pacote SPSS 17 (versão demonstrativa).

\section{Resultados}

Foram determinadas as médias de pontuação entre o AVD Katz e o CDR. A média é o valor que aponta para onde mais se concentram os dados de uma distribuição e do desvio padrão (D.P.) é uma medida de dispersão da amostra (BARBETTA et al., 2004). O escore varia de zero a três, dependendo do comprometimento cognitivo do avaliado. Esses pacientes tiveram os valores categóricos comparados com a pontuação no KATZ, como se pode observar na Tabela 2. 
Tabela 2 - Média e desvio padrão de grupo de indivíduos que realizaram tanto o AVD KATZ e o CDR

\begin{tabular}{c|c|c|c}
\hline $\begin{array}{c}\text { Idosos que fizeram o AVD } \\
\text { KATZ e o CDR }\end{array}$ & Pontuação no CDR & Média de pontuação no KATZ & D.P. \\
\hline 6 & 0 & 5,75 & 0,27 \\
16 & 0,5 & 5,78 & 0,31 \\
26 & 1 & 5,00 & 1,31 \\
12 & 2 & 4,50 & 1,19 \\
1 & 3 & 0,50 & \\
\hline
\end{tabular}

A Tabela 3 mostra os valores obtidos no CDR correlacionados com os resultados dos pacientes agrupados em seus respectivos pontos no AVD Katz.

Tabela 3 - Correlação entre os escores do teste CDR e seus respectivos grupos no AVD Katz avaliado pelo coeficiente de correlação de Pearson

\begin{tabular}{c|c|c|c}
\hline CDR & $\mathrm{N}$ & $\mathrm{Z}$ & $p$ \\
\hline 0 e 0,5 & 22 & $-0,384$ & $\mathrm{p}>0,05$ \\
0 e 1 & 32 & $-1,580$ & $\mathrm{p}>0,05$ \\
0 e 2 & 18 & $-2,023$ & $\mathrm{p}>0,05$ \\
0,5 e 1 & 42 & $-2,571$ & $\mathrm{p}<0,01$ \\
\hline
\end{tabular}

A medida de maximização da expectativa para calibrar os parâmetros dos itens foi realizada pelo Xcalibre. O processo de estimativa é iterativo e repete-se em ciclos (loops) até que o critério de convergência seja atingido. O pequeno número de ciclos necessários foi evidência para o ajuste dos dados ao modelo da TRI (pressuposto da unidimensionalidade). O AVD KATZ teve 22 loops, o que indica que está dentro dos padrões (abaixo de 50 ciclos é o ideal).
A calibração foi feita a partir do Modelo Generalizado de Crédito Parcial (Generalized Partial Credit Model - GPCM), e o método de estimação do teta foi a Estimação Máxima de Probabilidade (Maximum Likelihood Estimation - MLE) e o critério de convergência foi de 0,01 .

A Figura 1 mostra a distribuição do teta calibrado de todos os itens do teste. Nota-se que, nesse teste, o teta ficou entre zero e $-2,5$; o que significa que a amostra utilizada possui competência de média à baixa.

Figura 1 - Teta estimado dos pacientes

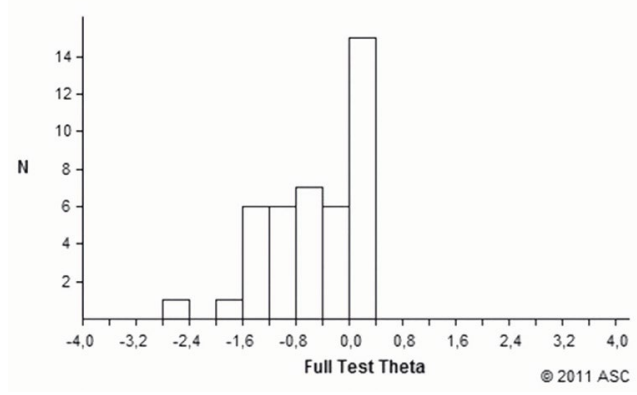


O parâmetro a de todos os itens calibrados teve média de $2,53(0,406)$. $\mathrm{O}$ alfa do teste (contendo os seis itens) foi de 0,892. A Figura 2 mostra a Função de Informação do Teste (FIT), que seria uma representação de quanta informação o teste fornece em cada nível de teta. $\mathrm{O}$ teste oferece mais informação entre os tetas -1 a -2. A Figura 3 mostra a função de Condição de Medida de Erro Padrão (CMEP), que é inversa à função FIT e estima a quantidade de erro estimado de teta para cada nível. O erro é menor entre os valores de teta entre 0 e -2 .

Figura 2 - Função de informação do teste (FIT) do AVD KATZ

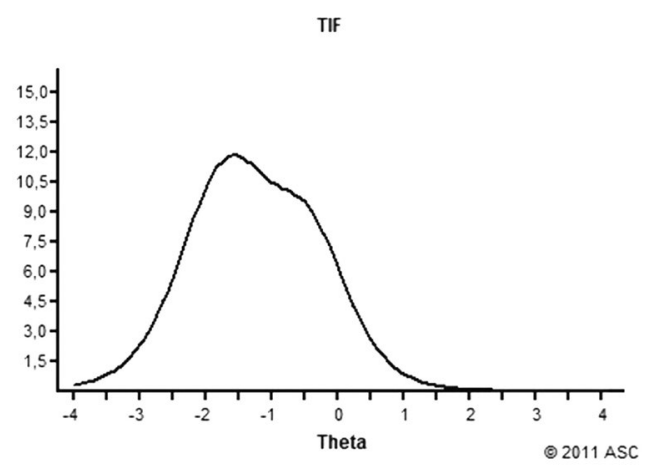

Figura 3 - Condição de medida de erro padrão (CMEP) do AVD KATZ

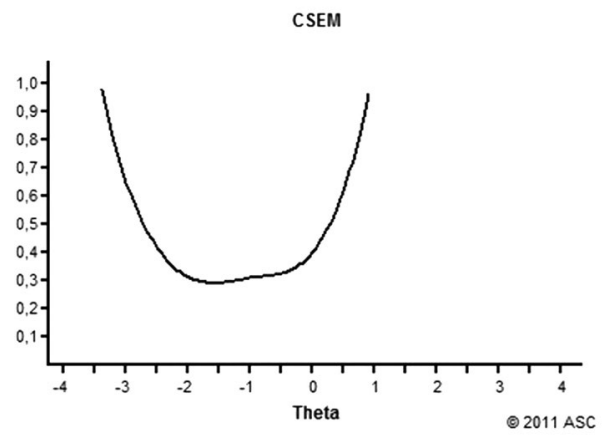

A Tabela 4 apresenta um sumário estatístico para o item. Assim, temos a correlação $r$, que é a correlação de Pearson do item com o teta estimado. $\mathrm{O}$ $r$ varia de -1 a 1 , com maior valor indicando maior discriminação. $\mathrm{O}$ alfa w/o é o alfa de Cronbach computado com o item excluído. A correlação total do item é a medida do poder de discriminação do item e o parâmetro da TRI relacionado a ele (Tabela 4). O parâmetro a indica a discriminação do item, como citado anteriormente. Um alto valor nesse índice resulta em um grau de inclinação ainda maior da TRI e indica que o item também diferencia os examinandos.

Tabela 4 - Itens do AVD KATZ correlacionados ao total do teste e à dificuldade de realização de cada um

\begin{tabular}{l|c|c|c|c|c}
\hline \multicolumn{1}{c|}{ Item } & Correlação $r$ & Parâmetro a & e.p. & Chi quadrado & Alfa w/o \\
\hline Higiene & 0,649 & 2,736 & 0,694 & 2,674 & 0,864 \\
Vestir-se & 0,715 & 2,703 & 0,703 & 4,534 & 0,870 \\
Banheiro & 0,518 & 2,768 & 0,710 & 2,761 & 0,863 \\
Locomoção & 0,595 & 2,206 & 0,567 & 9,455 & 0,881 \\
Continência & 0,612 & 1,860 & 0,500 & 5,655 & 0,902 \\
Alimentação & 0,646 & 2,908 & 0,730 & 5,123 & 0,857 \\
\hline
\end{tabular}


O parâmetro b indica o limiar do item e indica a localização do teta numa série em que a probabilidade da resposta equivale a $50 \%$. Um alto grau nos erros padrões e.p. indica que o parâmetro do item foi insuficientemente estimado. A Tabela 5 mostra a dificuldade encontrada em passar da dependência total para a dependência assistida e dessa para a independência total nos determinados itens do AVD Katz.

Tabela 5 - Parâmetro b (limiar) de dificuldade em passar de um estado de total dependência (0) para dependência assistida $(0,5)$ e deste para autonomia total (1)

\begin{tabular}{l|r|r}
\hline \multicolumn{1}{c|}{ Item } & $\mathrm{b}(0 \mathrm{a}$ 0,5) & $\mathrm{b}(0,5 \mathrm{a} 1)$ \\
\hline Higiene & $-1,749$ & $-0,490$ \\
Vestir-se & $-1,630$ & $-0,208$ \\
Banheiro & $-1,786$ & $-1,229$ \\
Locomoção & $-1,991$ & $-0,702$ \\
Continência & $-2,372$ & $-0,573$ \\
Alimentação & $-2,158$ & $-0,568$ \\
\hline
\end{tabular}

A Figura 4 representa geometricamente a probabilidade de pertinência do paciente com determinado nível de teta à categoria um (equivalente a zero, que significa total dependência), categoria dois (equivalente a 0,5 que é a dependência assistida) e categoria três (equivalente a um, total independência).
Figura 4 - Curvas características dos itens do AVD KATZ

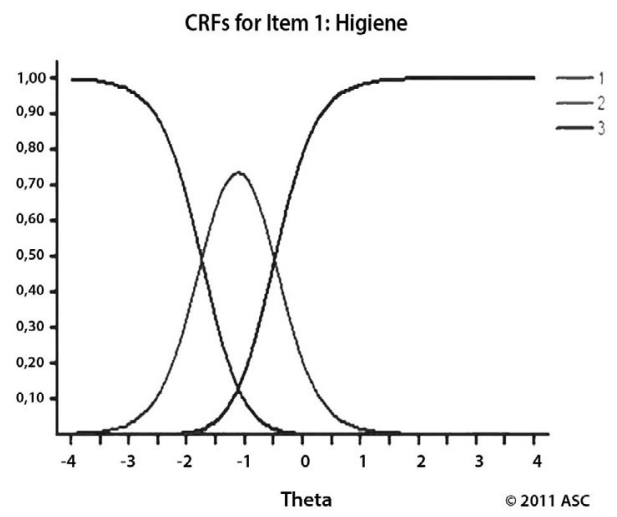

CRFs for Item 2: Vestir

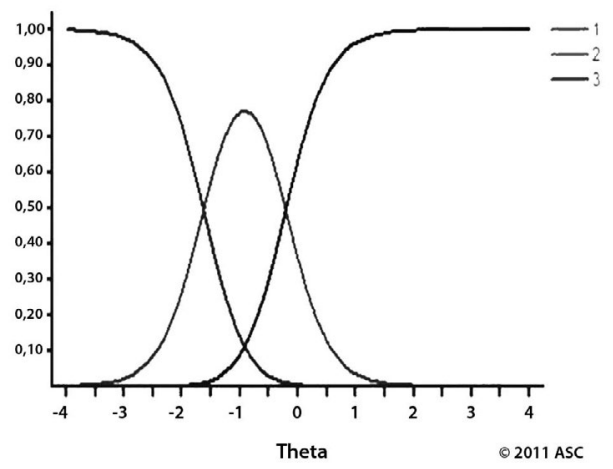

CRFs for Item 3: Banheir

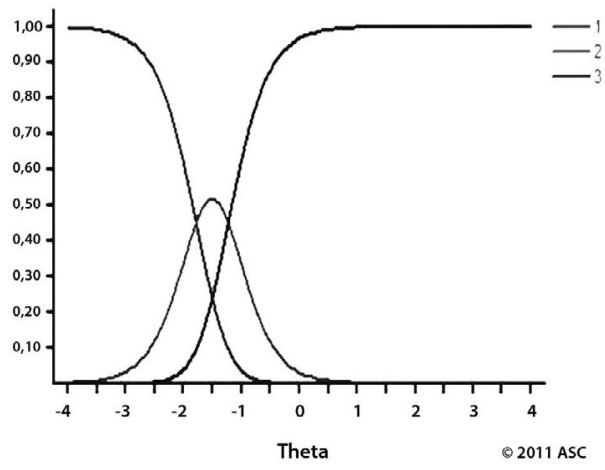




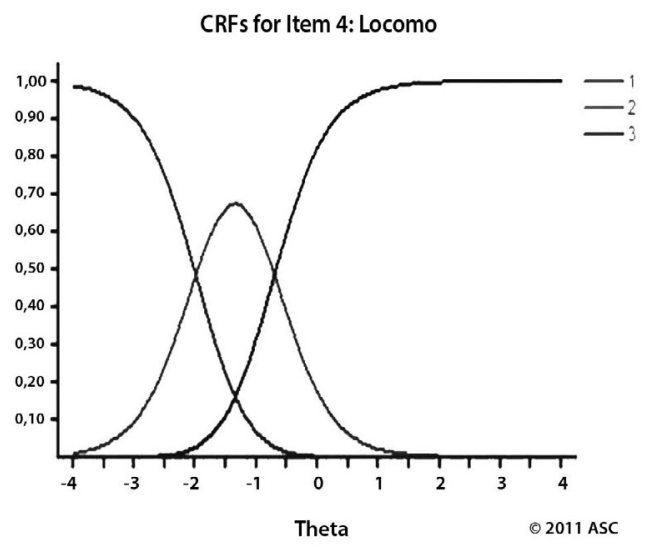

CRFs for Item 5 : Contin

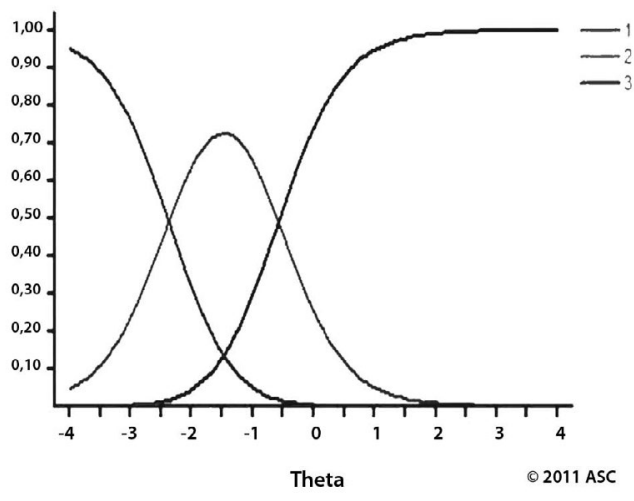

CRFs for Item 6: Aliment

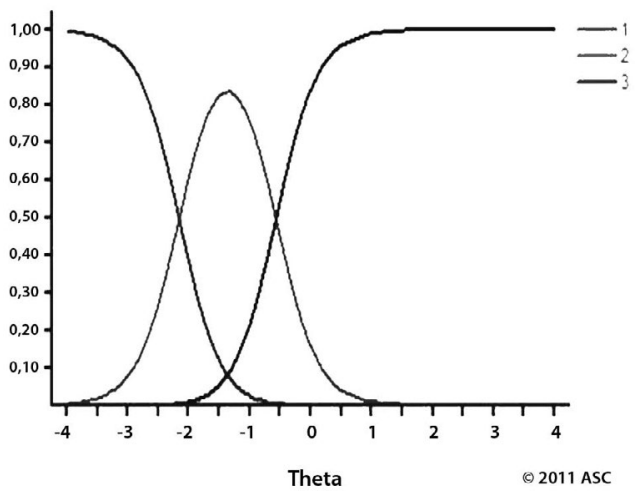

Por fim, a Tabela 6 mostra a frequência de pacientes que responderam a cada categoria dos itens do instrumento.

Tabela 6 - Número e porcentagem de pacientes que marcaram cada item

\begin{tabular}{l|c|r|c}
\hline \multicolumn{1}{c|}{ Item } & \multicolumn{1}{c|}{0} & \multicolumn{1}{c|}{0,5} & 1 \\
\hline Higiene & $5(7,5 \%)$ & $18(26,9 \%)$ & $44(65,7 \%)$ \\
Vestir-se & $6(9,0 \%)$ & $23(34,3 \%)$ & $38(56,7 \%)$ \\
Banheiro & $4(6,0 \%)$ & $7(10,7 \%)$ & $56(83,6 \%)$ \\
Locomoção & $4(6,0 \%)$ & $16(23,9 \%)$ & $47(70,1 \%)$ \\
Continência & $3(4,5 \%)$ & $20(29,9 \%)$ & $44(65,7 \%)$ \\
Alimentação & $3(4,5 \%)$ & $18(26,9 \%)$ & $46(68,7 \%)$ \\
\hline
\end{tabular}

\section{Discussão}

Muitos estudos, como o de Thomas et al. (1998), utilizam as relações entre o CDR e os testes que avaliam a capacidade funcional, no caso deste trabalho o AVD Katz. Segundo esses autores, existe uma relação entre a gravidade das alterações cognitivas e a autonomia nas atividades diárias. As médias mostram que os pacientes avaliados estão com certo nível de autonomia preservado e o desvio padrão mostra que a variação dessas médias é pequena em relação à amostra.

Um dado interessante a ser avaliado na distribuição da amostra é a maioria de mulheres em relação a homens. Isso é comprovado na literatura, pois as mulheres, em grande parte, fazem uso dos serviços de saúde relacionado a questões reprodutivas (LAMBREW, 2002). Entretanto, existem outros fatores que também influenciam nesse dado: mulheres tendem a avaliar seu estado de saúde de forma mais negativa e também mencionarem mais doenças crônicas do que os homens, que, por sua vez, apresentam doenças comparativamente mais severas 
e de maior letalidade (VERBRUGGE; WINGARD, 1987).

De acordo com as análises estatísticas clássicas e com a TRI, o AVD Katz se mostrou uma avaliação útil com itens bastante informativos para a população alvo, o que mostra que ele é um bom instrumento para avaliação na capacidade preditiva da autonomia ou da dependência do paciente. Todos os itens discriminam muito bem os pacientes, em especial o de alimentação que teve um dos mais altos escores nessa categoria. $\mathrm{O}$ item continência possui a mais baixa discriminação, mas, mesmo assim, possui uma boa correlação com o escore total do teste.

$\mathrm{O}$ parâmetro de dificuldade $\mathrm{b}$ mostrou que é mais fácil passar da dependência para a dependência assistida do que passar dessa para a autonomia completa em todos os itens. A grande maioria dos pacientes avaliados tinham boa independência na realização das tarefas. A maior dependência foi notada no item vestir-se com 6 (9\%) totalmente dependentes, 23 (34,3\%), necessitando de ajuda e apenas $38(56,7 \%)$ realizando a tarefa por completo. $\mathrm{O}$ item banheiro teve o maior índice de autonomia, com $56(83,6 \%)$ dos participantes do estudo respondendo à categoria 1 . Podemos considerar que esse item, assim como Alimentação, são as tarefas mais importantes ao ser humano (considerando sua filogenia), sendo, portanto, mais preservadas ao longo da vida do indivíduo. Estudos como o de Gill et al. (1996) mostram que prejuízos nos desempenhos físicos e cognitivos contribuem de forma independente para o risco de dependência funcional em idosos.
Outros estudos mostram que cerca de $40 \%$ dos indivíduos com 65 anos ou mais de idade precisam de algum tipo de ajuda para realizar pelo menos uma tarefa instrumental de vida diária, e uma parcela menor $(10 \%)$ requer auxílio em atividades de vida diária (KARSCH, 2003).

O AVD Katz se mostrou bem sensível na discriminação entre pacientes com prejuízo cognitivo (escore 0,5 no teste CDR) e estágio inicial do processo demencial (escore 1 no teste CDR). Quanto menor for a pontuação no teste AVD Katz, maior será o comprometimento cognitivo do paciente e maior a chance de isto ser detectado caso os dois instrumentos sejam usados conjuntamente.

\section{Conclusão}

Nos anos 50, muitos cientistas passaram a concentrar seus esforços no desenvolvimento de instrumentos e de medidas que adicionassem significado e precisão quantitativa à magnitude e à gravidade dos problemas funcionais dos idosos. As teorias utilizadas com base na avaliação funcional de idosos não era satisfatória e as dimensões usadas de um instrumento a outro dificultava a comparação dos estudos. Por não serem precisas, as informações obtidas por meio desses instrumentos não eram adequadas para a realização de prognósticos e tomadas de decisões sobre tratamentos ou cuidados (KATZ; STROUD, 1987).

Com base em dados de um hospital em Cleveland, Ohio, Katz e Chinn (1951) desenvolveram um instrumento que buscava avaliar a independência funcional dos pacientes para banhar-se, 
vestir-se, ir ao banheiro, locomover-se da cama para a cadeira e vice-versa, ser continente e alimentar-se. Para isso, registrava-se o tipo de assistência que o idoso recebia em cada uma das tarefas. Com base nas informações empíricas e teóricas, desenvolveram o Index of $A D L$ (Index of Activity Daily Living) usado para mensurar essas seis funções.

Por ser um teste relativamente antigo (KATZ et al., 1963) e ainda utilizado em âmbito nacional e internacional, uma revisão e readaptação se faz necessária. $\mathrm{Na}$ época, essa era a vida básica de um idoso, um ciclo entre alimentar-se, locomover-se, vestir-se, despir-se para ir ao banheiro e evacuar. Hoje em dia, no Século XXI, a chamada terceira idade está muito mais dinâmica e não apenas espera por seu leito de morte. Com o advento da inclusão digital qualquer um pode utilizar da tecnologia disponível a preços acessíveis e facilidade de uso.

Nota-se aqui que a escolaridade da amostra é muito baixa, mais de um terço era analfabeta e apenas sete pessoas possuíam mais de doze anos de estudo. Segundo a Pesquisa Nacional por Amostra de Domicílios (PNAD) do IBGE entre pessoas com dez anos, a taxa de analfabetismo é de $9 \%$ e a meta é chegar a 6,7\% em 2015, como exige a Organização das Nações Unidas para a educação, a ciência e a cultura (Unesco). Esse dado pode influenciar em uma nova avaliação com uma amostra de pessoas com mais anos de estudos no futuro.

\section{Item response theory applied to the inventory of daily living of Katz in a sample of the Regional Hospital of Asa Norte - Brasília/DF}

\section{Abstract}

This paper will analyze the Inventory of Daily Living Katz (Katz IDL) from statistical analysis using Item Response Theory (IRT). Some studies show that dementia is linked to a genetic susceptibility and causes damage to the functional activities of the individual and, therefore, the importance of functional assessment of the elderly. For that, there are reviews of Daily Living which measure the basic functions of individual autonomy. The TRI can replace, in parts, the Classical Test Theory (CTT), because it assesses whether the item actually measures the latent trait that should be measured. The study objectives were to assess whether the items actually measured the Katz IDL autonomy; analyze the responses of patients and verify discrimination and difficulty of each item. The sample, collected from the Regional Hospital Asa Norte (HRAN) Brasília/DF, had a total of 67 patients, $42(62.7 \%)$ women and $25(37.3 \%)$ men. From the analysis made with the Xcalibre software, can be noted that the Katz IDL test has good sensitivity in differentiating patients with cognitive impairment and early dementia, according to the score of the Clinical Dementia Rating (CDR). Moreover, proved to be a test that measures the patient's autonomy in relation to its activities in life and your items have good correlation with the total test. The test has good validity today, but in the future, with better education and the advent of technology available to everyone, some items may be subject to change.

Keywords: Funcional Evaluation. Dementia. Item response theory (IRT). Inventory of daily activities. 


\section{Referências}

ANDEL, R.; CROWE, M.; PEDERSEN, N. FRATIGLIONI, L.; JOHANSSON, B.; GATZ, M. Physical exercise at midlife and risk of dementia three decades later: a population-based study of swedish twins. Journal of Gerontology, Washington, v. 63, n. 1, p. 62-66, Jan. 2008.

BARBETTA, P. A.; REIS M.M.; BORNIA A.C. Estatística para cursos de Engenharia e Informática. São Paulo: Atlas, 2004.

BERG, L. Clinical Dementia Rating (Correspondence). Psychiatry, Condon, v. 145, p. 339, Sep. 1984.

CAIXETA, L. Velho Mal do Novo Milênio. Revista de psicologia, psicanálise, neurociência e conhecimento, São Paulo, v. 172, p. 76-83, maio 2007.

CORAZZA, M. A. Terceira Idade e Atividade Física. São Paulo: Phorte, 2001.

DUARTE, Y; ANDRADE, C.; LEBRÃO, M. L. O Índice Katz na avaliação da funcionalidade dos idosos. Revista da Escola de Enfermagen da USP, São Paulo, v. 41, n. 2, p. 317-325, ago. 2007.

FRATIGLIONI, L.; GRUT, M.; FORSELL, Y.; VIITANEN, M.; GRAFSTRÖM, M.; HOLMEN, K. Prevalence of Alzheimer disease and other dementias in an elderly urban population: relationship with age, sex, and education. Neurology, United States, v. 41, p. 1886-1892, Dec. 1991.

FREITAS, E. V. Demografia e epidemiologia do envelhecimento. In: Ligia, P. Y. et al. (Orgs.). Tempo de Envelhecer: percursos e dimensões psicossociais. Rio de Janeiro: NAU Editora, 2004. p. 257-283

GILL, T.M.; WILLIAMS, C. S.; RICHARDSON, E. D.; TINETTI, M. E. Impairments in physical performance and cognitive status as predisposing factors for functional dependence among nondisabled older persons. Journal of Gerontology, Washington, v. 51, n. 6, p. 283-288, Nov. 1996.
HAMBLETON, R. K.; SWAMINATHAN, H.; ROGERS, H. J. Fundamentals of item response theory. Newbury Park, California: SAGE Publications, 1991.

IBGE (Fundação Instituto Brasileiro de Geografia e Estatística). Dados sobre População do Brasil, PNAD (Pesquisa Nacional por Amostra de Domicílios) 2010. Disponível em: <http://www.ibge.gov.br/estadosat/perfil. php? sigla $=\mathrm{df}>$. Acessado em: 10 jul. 2010.

JORM, A. F.; JACOMB, P. A. The informant questionnaire on cognitive decline in the elderly (IQCODE): Sociodemographic correlates, reliability, validity and some norms. Psychological Medicine, Cambridge, v. 19, p. 1015-1022, Nov. 1989.

KAARLOLA, A.; TALLGREN, M.; PETTILA, V. Long-term survival, quality of life and quality-adjusted life-years among critically ill elderly patients. Critical Care Medicine, Mount Prospect, v. 34, n. 8, p. 2120-2126, Aug. 2006.

KARSCH, U.M. Idosos dependentes: famílias e cuidadores. Cadernos de Saúde Pública, Rio de Janeiro, v. 19, n. 3, p. 861-866, maio/jun. 2003.

KATZ, S.; AKPOM, C.A. A measure of primary sociobiological functions. International Journal of Health Services, New York, v. 6, n. 3, p. 493-508, Feb. 1976.

KATZ, S.; CHINN, A. B. Multidisciplinary studies of illness in aged persons II: a new classification of functional status in Activities of Daily Living. Journal of Chronic Diseases, Oxford, v. 9, n. 1, p. 55-62, Jan. 1959.

KATZ, S; FORD, A. B.; MOSKOWITZ, R. W.; JACKSON B. A.; JAFFE M. W. Studies of illness in the aged. The index of ADL: a standardized measure of biological and psychosocial function. Journal of the American Medical Association, Chicago, v. 185, n. 12, p. 914-919, Sep. 1963.

KATZ, S.; STROUD, M. W. Functional assessment in geriatrics: a review of progress and directions. Journal of the American Geriatric Society, New York, v. 37, n. 3, p. 267-271, Mar. 1989. 
LAMBREW, J. M. Diagnosing disparities in health insurance for women: a prescription for change. Disponível em: <http://www. commonwealthfund.org/publications/fundreports/2001/aug/diagnosing-disparities-inhealth-insurance-for-women--a-prescriptionfor-change>.pdf. Acesso em: 30 set. 2001.

LAWTON, M. P.; BRODY, M. H. Assessment of older people: self-maintaining and instrumental activities of daily living. The Gerontologist, Oxford, v. 9, n. 3, p. 179-186, Aug. 1969.

LOEVINGER, J. A systematic approach to the construction and evaluation of tests of ability. Psychological Monographs, Washington, v. 61, n. 4, p. 1-49, Jun. 1947.

MCHORNEY, C.; COHEN, A. Equating health status measures with Item Response Theory: Illustrations with functional status. Medical Care, West Chester, v. 39, Suppl. 9, p. 43-59, Sep. 2000.

OKUMA, S. S. $O$ idoso e a atividade física: fundamentos e pesquisa. Campinas-SP: Papirus, 1998.

PACHECO, R. O.; SANTOS, S. S. Avaliação Global de idosos em unidades de PSF. Textos Envelhecimento, Rio de Janeiro, v. 7, n. 2, p. 45-61, set. 2004.

PASQUALI, L. Teoria de Resposta ao Item: Teoria, Procedimentos e Aplicações. Brasília: LabPAM/UnB, 2007.

SCAZUFCA, M. Brazilian version of the Burden Interview scale for the assessment of burden of care in carers of people with mental illnesses. Revista Braileira de Psiquiatria, Rio de Janeiro, v. 24, n. 1, p. 12-17, 2002.

SCHOUERI JÚNIOR, R.; RAMOS, L. R.; PAPALÉO, M. N. Crescimento populacional: aspectos demográficos e sociais. In: Carvalho Filho, E.T., Papaléo, M. N. Geriatria: fundamentos clínica e terapêutica. São Paulo: Atheneu, 2000. p. 9-29.
SPECTOR, W.D.; FLEISHMAN J.A. Combining Activities of Daily Living With Instrumental Activities of Daily Living to Measure Functional Disability. Journal of Gerontology, Washington, v. 53, n. 1, p. 4657, Jan. 1998.

THOMAS, V. S.; ROCKWOOD, K.; MCDOWELL, I. Multidimensionality in instrumental and basic activities of daily living. Journal of Clinical Epidemiology, New York, v. 51, n. 4, p. 315-321, Apr. 1998.

VERAS, R. P.; RAMOS, L. R.; KALACHE, A. Crescimento da população idosa no Brasil: transformações e consequências na sociedade. Revista de Saúde Pública, São Paulo, v. 21, n. 3, p. 225-33, Jun. 1987.

UNESCO. Relatório de monitoramento de educação para todos Brasil 2008: educação para todos em 2015, alcançaremos a meta? Brasília: UNESCO, 2008.

VERBRUGGE, L. M.; WINGARD, D. L. Sex differentials in health and mortality. Women Health, New Rochelle, v. 12, n. 2, p. 103-145, Apr. 1987.

WILKINS, S.; LAW, M.; LETS, L. Assessment of functional performance. In: Bonder, B. R.; Wagner, M. B. Functional performance in older adults. Philadelphia: F. A. Davis, 2001. p. 236-251. 